\title{
Assessment of bone in Ehlers Danlos syndrome by ultrasound and densitometry
}

\author{
A L Dolan, N K Arden, R Grahame, T D Spector
}

\begin{abstract}
Objective-Ehlers Danlos syndrome (EDS) is an inherited disorder of connective tissue characterised by hyperextensible skin, joint laxity, and easy bruising. There are phenotypic similarities with osteogenesis imperfecta, but in EDS a tendency to fracture or altered bone mass has not previously been considered to be a cardinal feature.

Method-This case-control design study investigates whether 23 patients with EDS had differences in fracture rates, bone mass, and calcaneal ultrasound parameters compared with age and sex matched controls.
\end{abstract}

Results-23 cases of EDS (mean (SD) age 38.5 (15.5)) were compared with 23 controls (mean age $37.8(14.5)$ ). A significant reduction in bone density measured by dual energy $x$ ray absorptiometry was found at the neck of femur by $0.9 \mathrm{SD}, \mathrm{p}=$ 0.05 , and lumbar spine by $0.74 \mathrm{SD}, \mathrm{p}=$ 0.02 . At the calcaneum, broad band ultrasound attenuation and speed of sound were significantly reduced compared with controls by $0.95 \mathrm{SD}(\mathrm{p}=0.004)$ and 0.49 SD $(p=0.004)$ for broad band ultrasound attenuation and speed of sound respectively. Broad band ultrasound attenuation and speed of sound remained significantly reduced after adjusting for bone mineral density (BMD). After adjusting for functional status (HAQ), age and sex, hypermobility was inversely correlated with broad band ultrasound attenuation and SOS, but not BMD at hip or spine. Previous fracture was 10 times more common in EDS $(p<0.001)$, with $86.9 \%$ of patients reporting a total of 47 low impact fractures, compared with $8.7 \%$ of controls.

Conclusion-This study has identified a tendency of EDS patients to fracture, have low bone mass and abnormal bone structure. The aetiology is likely to be multifactorial, with an inherited structural element, accentuated by immobility or reduced exercise. This is one of the first clinical studies to suggest ultrasound can detect structural differences in bone, independent of dual energy $x$ ray absorptiometry.

(Ann Rheum Dis 1998;57:630-633)

Guys and St Thomas's

Hospital, London

Correspondence to:

Dr AL Dolan, Department

of Rheumatology, Greenwich

District Hospital, Vanburgh

Hill, London SE10 9EH.

Accepted for publication 21 August 1998 disorder of connective tissue characterised by hyperextensible skin, joint laxity, and easy bruising. ${ }^{1}$ In the more common forms I, II, and III, the underlying genetic defect is unclear, but a recent study has suggested linkage with the COL 5A1 gene. ${ }^{2}$ In the rarer type IV EDS, where vascular fragility predominates, it is a result of deficiency of type III collagen. ${ }^{3}$

Osteoporosis is not one of the cardinal features of EDS and is not described in the early reports. ${ }^{4}$ However, osteopenia is reported in the very rare type VI EDS together with muscle hypotonia, kyphoscoliosis, and rupture of the ocular globe. ${ }^{1}$ Two recent short reports on four and seven patients respectively, who were identified through osteoporosis screening, have suggested that bone density may be reduced in EDS, ${ }^{56}$ although a small sample size and potential referral and selection bias limit the interpretation of these findings.

To our knowledge, this is the first systematic study of bone density in EDS. It uses a case-control design to investigate whether patients with EDS have differences in fracture rates, bone mass and heel ultrasound parameters compared with age and sex matched controls.

\section{Methods}

SUBJECTS

Twenty three patients ( 5 male, 18 female) with EDS were recruited from the EDS patient support group and from the rheumatology outpatient clinic at Guy's Hospital, most had been recruited during a previous cardiac study unrelated to this hypothesis. All gave informed consent. Their mean age was 38.5 years (range 18-64). All were white. Three women were post-menopausal (1, 2, and 10 years). The diagnosis of EDS was confirmed in all patients on the basis of hypermobile joints, hyperextensible skin, fragile tissues, and a tendency to form papyraceous scars and the identification of subtypes was according to the Berlin Nosology. ${ }^{7}$ Twenty three normal controls matched by sex, age, race, and menopause status were obtained from hospital and university staff. No patients or controls were taking corticosteroids, receiving hormone replacement therapy, bisphophonates, calcium or other bone modifying treatments.

STUDY DESIGN

All subjects completed a standardised questionnaire $^{8}$ to establish the lifetime incidence, site, and impact of fractures. Only low trauma fractures from a standing height or less were included. Each completed a demographic questionnaire as to environmental risk factors for osteoporosis. Disability was assessed by the Health Assessment Questionnaire. ${ }^{9}$ Hypermobility was assessed by the nine site Beighton score $^{10}$ and the Contompasis score, which 
Table 1 Clinical characteristics

\begin{tabular}{llll}
\hline & Cases $(n=23)$ & Controls $(n=23)$ & $p$ Value \\
\hline age (mean/SD) & $38.5(15.5)$ & $37.8(14.5)$ & 0.86 \\
sex (female) & $17 / 23$ & $17 / 23$ & $\mathrm{~ns}$ \\
smoking (yes/no) & 5 & 2 & $\mathrm{~ns}$ \\
alcohol (units/week) & $3.37(5.5)$ & $8.08(5.5)$ & 0.004 \\
height (cm) (mean/SD) & $166.6(9.8)$ & $169.69(7.9)$ & 0.25 \\
weight (kg) (mean/SD) & $65.52(12.2)$ & $66.78(11.5)$ & 0.71 \\
Contompasis (median/range) & $30.5(28.5,37.5)$ & $18(18,19)$ & $<0.001$ \\
Beighton (median/range) & $5(4,7)$ & $0(0,1)$ & $<0.001$ \\
HAQ (median/range) & $0.75(0.13,1.9)$ & $0(0,0)$ & $<0.001$ \\
\hline
\end{tabular}

Table 2 Regression of joint hypermobility with $B U A$, $S O S$, and $B M D^{\star}$

\begin{tabular}{llll}
\hline Beighton $v$ & $b$ & $r$ & $p$ Value \\
\hline BUA & -2.44 & -0.36 & 0.02 \\
SOS & -5.44 & -0.30 & 0.09 \\
BMD lumbar spine & -0.003 & -0.28 & 0.53 \\
BMD femoral neck & -0.004 & -0.19 & 0.39 \\
Contompasis v & & & \\
BUA & -0.72 & -0.33 & 0.02 \\
SOS & -2.00 & -0.31 & 0.03 \\
BMD lumbar spine & 0.0008 & -0.22 & 0.65 \\
BMD femoral neck & 0.0006 & -0.11 & 0.38 \\
\hline
\end{tabular}

*All regressions adjusted for age, sex, and HAQ score. Units: $\mathrm{BMD}$ in $\mathrm{g} / \mathrm{cm}^{2}$, BUA in $\mathrm{dB} / \mathrm{MHz}$, SOS in $\mathrm{m} / \mathrm{s}$.

provides a more quantitative score of the same nine joint movements with a maximum score of 52. ${ }^{11}$

Bone mineral density (BMD), expressed in $\mathrm{g} / \mathrm{cm}^{2}$, was measured by dual energy $x$ ray absorptiometry (DXA) using a Hologic QDR 2000, at the left hip at the femoral neck, trochanter and lumbar spine L1-4. Coefficient of variation $(\mathrm{CV})$ was $0.6-1.6 \%$ from our own repeated measure on 70 patients. Broad band attenuation (BUA), in $\mathrm{dB} / \mathrm{MHz}$ and speed of sound (SOS), in $\mathrm{m} / \mathrm{s}$ were measured at the calcaneus using an ultrasound densitometer (Cuba McCue) $\mathrm{CV}=2.5 \%$ and $0.44 \%$ respectively). All z scores quoted are with respect to the manufacture's database.

\section{STATISTICS}

Dichotomous variables were compared using McNemar's $\chi^{2}$ test and continuous variables using paired $t$ test. Adjustments for BMD, $\mathrm{HAQ}$, age, and sex were made using multiple linear regression and paired $t$ tests performed on residuals from these analyses.

\section{Results}

The cases and controls were well matched for age, sex, height, weight, and current smoking

Table 3 Fractures, BMD, and ultrasound in Ehlers Danlos patients and normal controls

\begin{tabular}{llll}
\hline & Ehlers-Danlos & Controls & p Value \\
\hline Fracture (y/n) & $18 / 23$ & $3 / 23$ & $<0.001$ \\
BMD lumbar spine & $0.991(0.104)$ & $1.06(0.110)$ & 0.02 \\
BMD femoral neck & $0.795(0.1)$ & $0.851(0.084)$ & 0.05 \\
BMD trochanter & $0.657(0.117)$ & $0.743(0.1)$ & 0.02 \\
BUA & $71.35(17.66)$ & $92.24(20.32)$ & 0.004 \\
SOS & $1636.9(71.9)$ & $1702(45.2)$ & 0.004 \\
SOS (FN) & $1431.27(70.4)$ & $1484.12(41.6)$ & 0.026 \\
SOS (LS) & $1516.12(72.3)$ & $1572.97(46.5)$ & 0.012 \\
BUA (FN) & $2.77(16.9)$ & $19.34(19.5)$ & 0.024 \\
BUA (LS) & $21.78(17.8)$ & $39.03(20.7)$ & 0.02 \\
BUA (HA) $\dagger$ & $81.91(17.9)$ & $92.40(20.18)$ & 0.15 \\
SOS (HAQ) & $1675(67.3)$ & $1703.4(44.4)$ & 0.19 \\
BMD LS (HAQ) & $1.033(0.102)$ & $1.065(0.110)$ & 0.31 \\
BMD FN (HAQ) & $0.844(0.091)$ & $0.851(0.086)$ & 0.81 \\
\hline
\end{tabular}

${ }^{\star} \mathrm{SOS}(\mathrm{FN})=$ SOS adjusted for BMD femoral neck. LS = lumbar spine. $+\mathrm{BUA}(\mathrm{HAQ})=\mathrm{BUA}$ adjusted for HAQ score. Values are presented as means (SD). Units: BMD in $\mathrm{g} / \mathrm{cm}^{2}$, BUA in $\mathrm{dB} / \mathrm{MHz}$, SOS in $\mathrm{m} / \mathrm{s}$. status, although the control group had a higher regular alcohol intake (table 1 ). The mean $\mathrm{z}$ scores, at hip and spine, of the control group were not significantly different from zero suggesting they were representative of the larger population in the database. The cases had higher joint hypermobility scores, as assessed by the median Beighton of 5 (range 4-7) and median Contompasis score of 30.5 (range 28.5-37.5) $(\mathrm{p}<0.001)$ and a disability score as assessed by HAQ of 0.75 (range 0.131.9) $(\mathrm{p}<0.001)$. Three were EDS type I, 7 type II, 13 type III.

There was a modest correlation between SOS and BMD of the femoral neck $(r=0.36$, $\mathrm{p}=0.02)$ and between BUA and BMD of the femoral neck for cases $(r=0.36, \mathrm{p}=0.02)$. The HAQ score was inversely correlated with BUA, SOS, and BMD of the femoral neck and lumbar spine $(r=-0.35$ to $-0.47, \mathrm{p}<0.05)$. After adjusting for $\mathrm{HAQ}$, age, and sex the extent of a patients hypermobility assessed by either scale was inversely correlated with BUA and SOS, but not BMD at either site (table 2). HAQ showed a weak inverse relation to hypermobility. (Beighton $r=-0.22$, Contompasis $r=$ $-0.28, \mathrm{NS})$.

We found a significant reduction in bone density by DXA at the two sites, hip (femoral neck), and the spine (table 3). BMD of femoral neck was reduced by 0.9 standard deviations (SD), $\mathrm{p}=0.05$, and lumbar spine by $0.74 \mathrm{SD}$, $\mathrm{p}=0.02\left(\right.$ mean BMD hip $=0.795(0.1) \mathrm{g} / \mathrm{cm}^{2}$, control BMD = $0.851(0.08) \mathrm{g} / \mathrm{cm}^{2} ; \mathrm{p}=0.05$; spine $\mathrm{BMD}=0.991(0.1) \mathrm{g} / \mathrm{cm}^{2}$, controls = $\left.1.06(0.1) \mathrm{g} / \mathrm{cm}^{2} ; \mathrm{p}=0.02\right)$. BUA at the calcaneum was significantly reduced by $0.95 \mathrm{SD}$, at 71.4 (17) $\mathrm{dB} / \mathrm{MHz}$ compared with 90.7 (19) $\mathrm{dB} / \mathrm{MHz}$ in controls $(\mathrm{p}=0.004)$. SOS was reduced with respect to controls by $0.49 \mathrm{SD}$ $($ EDS $=1637(72) \mathrm{m} / \mathrm{s}$, controls $=1702(45.2)$ $\mathrm{m} / \mathrm{s} ; \mathrm{p}=0.004)$. The differences in BUA and SOS remained significant after adjusting for $\mathrm{BMD}$ at the femoral neck or at the lumbar spine. The differences were in part explained by adjustment for functional status (HAQ) (table 3), which correlated with disease severity.

We found recalled fracture of at least one peripheral bone was 10 times more common in EDS than in controls $(\mathrm{p}<0.001)$. Twenty of $23(86.9 \%)$ patients had suffered a total of 47 low impact fractures, compared with two of 23 controls (8.7\%). No clinical vertebral fractures were reported. Fracture sites in EDS patients were as follows: 16 metatarsal, 10 Colles', 9 humerus, 7 fibula, 2 tibial, 2 femur, and one each of clavicle and scaphoid. Eleven of $47(23 \%)$ of fractures occurred in the second decade. The number of fractures was weakly correlated with HAQ ( $r=$ 0.25 , NS). There was no relation between the number of fractures and either score of hypermobility.

\section{Discussion}

This study shows that patients with EDS have a greater propensity to peripheral bone fractures than controls. There is a 0.89 SD reduction in hip BMD (femoral neck) and a 0.74 SD 
in spine $\mathrm{BMD}$, which would equate to approximately a twofold increase of fracture risk in a general population. There were also major independent reductions in calcaneal BUA and SOS, which are also associated with fracture risk. ${ }^{16-18}$ The classic osteoporotic fractures at hip and wrist are increased, with respect to controls, which had just one Colles'. However, the reported prevalence of all fractures in the EDS group, including sites not classically considered osteoporotic, was considerably greater than might be predicted from the reduction in BMD or ultrasound measures alone, which suggests the role of other factors, which we did not measure. Hypermobile joints have recently been associated with reduced joint proprioception. ${ }^{12}$ Increased body sway is a further risk factor for falls and thus fracture, ${ }^{13}$ which may have contributed to the increased fracture incidence.

Quantitative ultrasound is still being evaluated as a method of bone assessment. It is thought to give information about bone structure $^{1415}$ and predicts fracture independently of conventional bone densitometry. ${ }^{16-18}$ BUA is a measure of the attenuation of ultrasound through bone and is related to bone density, trabecular spacing, thickness, and connectivity. ${ }^{14}$ Speed of sound through a solid medium is related to the square of elasticity over density; as such it provides a measure of a medium's stiffness. In EDS we were able to demonstrate a significant $21 \%$ reduction in BUA with respect to controls. However, in common with many other ultrasound studies ${ }^{16}$ this did not correlate closely with DXA measures $(r=0.36)$, suggesting they may be measuring different properties of bone. Speed of sound was also reduced, although to a lesser extent. The reduction was greater for BUA than for BMD and there was a considerable independent component after adjusting for BMD, which suggests that ultrasound may be detecting changes in bone quality in EDS.

We have also demonstrated a significant correlation (table 2) between both ultrasound variables and the joint hypermobility score of a patient, such that those who are more hypermobile have reduced bone density and greater bone structural changes. This suggests that both skin and bone changes may be related to a common genetic defect. EDS patients may have an inherited defect in bone. EDS shows many phenotypic characteristics of osteogenesis imperfecta where the type 1 collagen defect leads to a quantitative reduction in collagen 1 fibres, ${ }^{19}$ however the propensity to fracture in EDS is reportedly present to a much lesser degree. Type 1 collagen has been excluded as a likely defect in EDS, but recent studies have suggested linkage to the COL 5A1 gene, which encodes the alpha $1(\mathrm{~V})$ chain of type $\mathrm{V}$ collagen in patients with type II EDS. ${ }^{2}$ Type V collagen is found in skin, cornea, and bone. It is has a fundamental role in fibrillogenesis, forming a core inside major collagen fibrils. ${ }^{20}$ Deficiency of such a molecule might result in misalignment of the collagen 1 molecule and so altered bone strength and tendency to fracture. EDS shares clinical features with the benign joint hypermobility syndrome and type III EDS and benign joint hypermobility syndrome may, indeed be one and the same. A recent study of BMD in benign joint hypermobility syndrome suggest a non-significant trend to reduced $\mathrm{BMD},{ }^{21}$ which may be explained by the presence of a less severe connective tissue disease seen in that patient group.

The differences in BMD, BUA, and SOS were reduced by adjusting for HAQ scores (table 3). This could suggest that a proportion of the difference in bone mass compared with controls could be accounted for by a patient's subsequent lack of mobility. However, this is likely to be an innate feature of the disease, rather than a secondary external confounding factor, although separating the different components may shed further light on the aetiology of bone loss in EDS. Immobility may have arisen from joint damage, deformity or muscle hypotonia. However, many of our patients had low bone mass even in their 20s, supporting an effect on bone mass that is either inherited or acting in the early years. EDS patients are often advised to avoid exercise and contact sport to avoid joint damage, bruising, and scarring. Reduced exercise in youth might result in a reduced peak bone mass; subsequent damage to hypermobile joints might further reduce their exercise tolerance with age.

This study has certain limitations. Cases are selected in part from a self help group and may not be representative of all patients with EDS. Selection bias could have occurred if those with the most severe disease or fractures preferentially volunteered. The subjects however were unaware of the hypothesis, having initially volunteered for a different study. Controls were selected from hospital staff to select a similar socioeconomic group and may have over represented a "well" group, but rates of fracture were similar to population data. ${ }^{8}$ There may be a recall bias in recall of fractures, with patients more likely to recall fractures, however the size of difference makes this unlikely. Other studies have shown recall of fractures to be reasonably accurate. ${ }^{8}$

There has been no formal study of the benefits of treatment of reduced bone density in EDS patients. Deodhar cited cases who did not respond to HRT. ${ }^{5}$ Use of HRT may be advantageous to limit further perimenopausal bone resorption. Intervention choices in premenopausal women and men are more difficult, with the role of diet exercise and drugs unproven. Even if the inherent defect does not respond to treatment, it is important to assess and intervene to limit any additive effect of other causes of bone loss, such as postmenopausal loss and patients with EDS should be made aware of this.

In summary, this study has shown that EDS patients have a previously unrecognised tendency to fracture characterised by a low bone mass and abnormal bone structure. This is likely to be multifactorial, with an inherited structural element, but accentuated by reduced mobility and the possibility of a proprioceptive defect. Patients with EDS should be investigated for osteoporosis and physical and 
pharmaceutical strategies to reduce fractures considered. This study also suggests that ultrasound can be useful in detecting structural differences in bone, independent of those detected by DXA.

1 Beighton P. The Ehlers Danlos syndrome.London: William Heinemann Medical Books, 1970.

2 Loughlin J, Irven C, Hardwick LJ, Butcher S, Walsh S, Wordsworth $\mathrm{P}$, et al. Linkage of the gene that encodes the Po type $\mathrm{V}$ collagen (COL5A1) to type II Ehlers Danlos syndrome. Hum Mol Genet 1995;4:164951.

3 Pope FM, Martin GR, Lichtenstein JR, Penttinen R, Gerson B, Rowe DW, et al. Patients with Ehlers Danlos syndrome type IV lack type III collagen. Proc Natl Acad Sci USA 1975;72:1314-16.

4 Beighton P, Horan F. Orthopaedic aspects of the Ehlers Danlos syndrome. J Bone Joint Surg 1969;51B:444-9.

5 Deodhar AA, Woolf AD. Ehlers Danlos syndrome and osteoporosis. Ann Rheum Dis 1994;53:841-2.

6 Coelho PC, Santos RA, Gomes JAM. Osteoporosis and Ehlers Danlos syndrome. Ann Rheum Dis 1994;53:212 13.

7 Beighton PH, dePaepe A, Danks D, Finidori G, GeddeDahl $\mathrm{T}$, et al. International nosology of heritable disorders of connective tissue, Berlin 1986. Am J Med Genet 1988;29:581-94.

8 Arden NK, Griffiths GO, Hart DJ, Doyle, DV, Spector TD. The association between osteoarthritis and osteoporotic
fractures: the Chingford study. Br J Rheumatol 1996;35: 1299-304.

9 Fritz JF, Spitz P, Kraines RG, Holman HR. Measurement of patient outcome in arthritis. Arthritis Rheum 1980;23: 352-64.
10 Beighton PH, Solomon L, Soskolne CL. Articular mobility in an African population. Ann Rheum Dis 1973;32:413-18.

11 McNerney JE, Johnston WB. Generalised ligamentous axity, hallux abductor valgus and the first metatarsocuneiform joint. J Am Podiatr Assoc 1979;69:69-82.

12 Mallik AK, Ferrell WR, McDonald AG, Sturrock RD. Impaired proprioceptive acuity at the proximal interphalangeal joint in patients with the hypermobility syndrome. Br J Rheumatol 1994;33:631-7.

13 Nguyen T, Sambrook P, Kelly P, Jones G, Lord S, Freund J, et al. Prediction of osteoporotic fractures by postural instability and bone density. BMJ 1993;307:1111-15.

14 Njeh CF, Boivin CM, Langton CM. The role of ultrasound in the assessment of osteoporosis. Osteoporosis Int 1997:7: $7-22$

15 Gluer CC, Wu CY, Jergas M, Goldstein SA, Genant HK. Three quantitative ultrasound parameters reflect bone structure. Calcif Tissue Int 1994:55:46-52.

16 Schott AM, Weill-Engerer S, Hans D, Duboeuf F, Delmas PD, Meunier PJ. Ultrasound discriminates patients with hip fracture equally well as dual energy x-ray absorptiometry and independently of bone mineral density. J Bone Miner Res 1995;10:243-9.

17 Gluer CC, Cummings SR, Bauer DC, Stone K, Pressman A, Mathur A, et al. Osteoporosis: association of recent fractures with quantitative ultrasound findings. Radiology 1996;199:725-32.

18 Turner Ch, Peacock M, Timmerman L, Neal JM, Johnston CC Jr. Calcaneal ultrasound measurements discriminate hip fracture independently of bone mass. Osteoporosis In 1995;5:130-5.

19 Kivirikko K. Collagens and their abnormalities in a wide spectrum of diseases. Ann Med 1993;25:113-26.

20 Fichard A, Kleman JP, Ruggiero F. Another look at collagen V and XI molecules. Matrix Biol 1995;14:515-31.

21 Mishra MB, Ryan P, Atkinson P, Taylor H, Bell J, Calver D, et al. Extra articular features of the benign hypermobility syndrome. B J Rheumatol 1996;35:861-6. 